$\Rightarrow$ EPIDEMIOLOGY

\section{Fickle fall predicts flu}

Influenza infections occur more frequently in the winter than summer (hence the term 'flu season'), because cold, dry weather conditions are favourable for transmission. As a result, it has been predicted that climate change, and the associated warmer temperatures, will decrease the spread of flu. However, the unusually warm winter of 2017-2018 saw a severe global flu outbreak, calling this logic into question.
Qi Liu and Zhe-Min Tan of Nanjing University, China, and colleagues investigated the link between weather variability and flu infection numbers from 1997 to 2018. They compared 'rapid weather variability' (RWV), which quantifies the number of consecutive days with at least $3 \mathrm{~K}$ temperature differences over a 3-week period, with the incidence of flu in mainland China, Europe, and the USA. The researchers found that high RWV during late

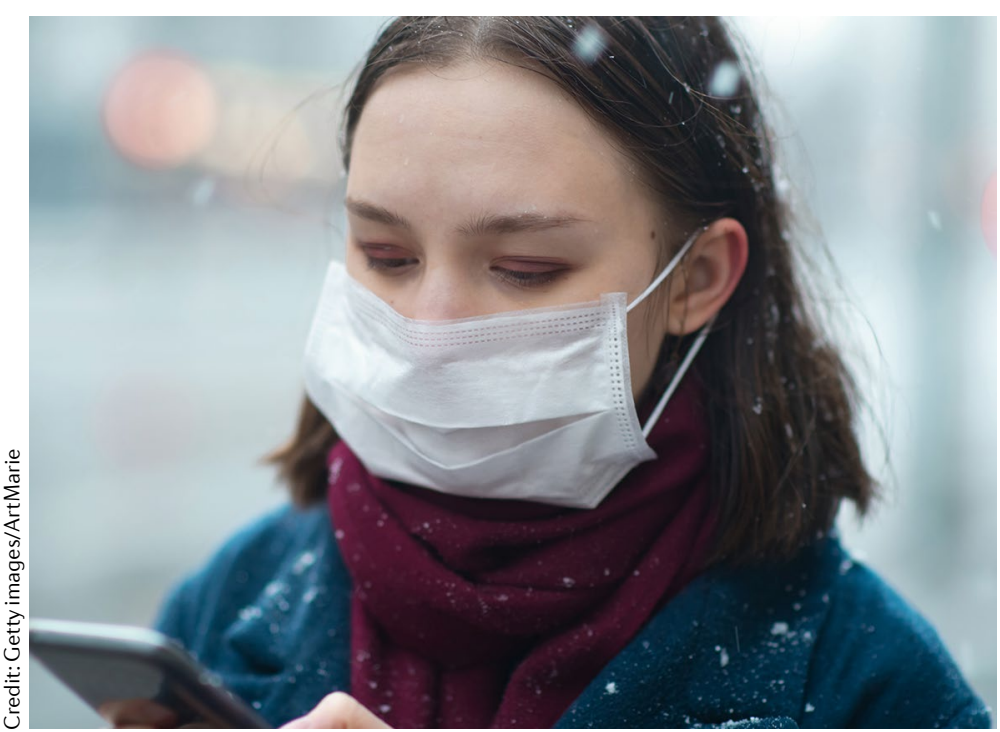

autumn is statistically linked to the severity of the flu outbreak later in winter. Potentially, the link is attributable to a weakening of the immune system following repeated temperature swings, specifically for the very young and old, leading to more infections and high rates of flu transmission in densely populated areas. CMIP5 simulations forced by RCP8.5 further indicate an increase in RWV, resulting in a modelled increase in infections in China, Europe and the populous north-eastern USA by up to $50 \%$. The predictions were less severe in the RCP4.5 scenario, estimating increases of up to $30 \%$.

While the connections between temperature and flu outbreaks are statistically significant, the analyses are mainly correlative and based on broad regional scales, with no consideration of other influencing factors such as age and immunization rates. However, as autumn temperature swings impact late winter outbreaks, the RWV in October and November could potentially be used as an early indicator of a severe flu season, giving health professionals more time to prepare and to minimize outbreaks.

Laura Zinke

ORIGINAL ARTICLE Liu, Q. et al. Changing rapid weather variability increases influenza epidemic risk in a warming climate. Environ. Res. Lett. https://doi.org/10.1088/1748-9326/ab70bc (2020) 